\title{
Remarques méthodologiques sur la participation du Québec à l'enseignement postsecondaire*
}

\author{
JACQUES DÉSY**
}

\section{RÉSUMÉ}

Selon un rapport du Secrétariat d'Etat (Quelques caractéristiques des étudiants du niveau postsecondaire au Canada - 1976), le Québec, suivi de l'Ontario, a le plus haut taux de participation à l'enseignement postsecondaire et toutes les autres provinces sont fortement sous-représentées. L'article différencie la participation selon le type d'étudiant (collégial, universitaire): au collégial, prépondérance du Québec; à l'universitaire, prépondérance accrue de l'Ontario, forte sous-représentation du Québec, différences moins marquées pour les autres régions. Les différences de structure, au collégial et à l'universitaire, entre le Québec et les autres régions canadiennes, ont un effet sur les taux de participation. La mesure de la participation du Québec à l'enseignement supérieur doit tenir compte des changements de structures scolaires survenus au Québec et distinguer les secteurs anglophone et francophone.

\section{ABSTRACT \\ Quebec Participation in Post-Secondary Education: Some Methodological Remarks}

According to a Secretary of State Report (Some Characteristics of Post-Secondary Students in Canada-1976), Quebec, followed by Ontario, had the highest post-secondary participation rate, and the other Provinces were highly under-represented. From the participation differentiation by type of student (college, university) that is presented here, it is suggested that: at the college level, Québec is well over-represented; at the university level, Ontario is more over-represented, Québec is highly under-represented, and the other regions show less marked differences. Participation rates may be affected by the different post-secondary systems of Québec and other Canadian regions. The estimation of Québec participation to

*Présenté à la réunion de la Société canadienne pour l'étude de l'enseignement supérieur, Congrès des sociétés savantes, University of New Brunswick, Fredericton, 11 juin 1977.

**Responsable de la recherche institutionnelle, Université du Québec à Trois-Rivières. 
higher education should take into account recent changes in Québec post-secondary structures and should differentiate between the francophone and the anglophone sector.

\section{INTRODUCTION}

A la demande du Secrétariat d'Etat, Statistique Canada a entrepris au début de 1975 un sondage auprès des étudiants du niveau postsecondaire au Canada. Les résultats de l'enquête qui portent sur 60000 réponses reçues, pondérées de manière à obtenir des estimations sur l'ensemble de la population étudiante, ont fait l'objet d'un rapport du Secrétariat d'Etat paru en $1976^{1}$.

Comme "l'enquête a été conçue dans le but de recueillir des données précises qui pourront servir à déceler et à analyser les écarts entre la participation à l'enseignement postsecondaire" ${ }^{2}$, il apparaît opportun de voir dans quelle mesure la conception de l'étude, les définitions utilisées ou les résultats du rapport permettent d'établir des comparaisons valides et fidèles du Québec et des autres régions canadiennes.

Dans cette perspective nous poursuivons la description de la participation régionale à l'enseignement postsecondaire en la différenciant selon le type d'étudiant ou le niveau d'études. Nous rappelons aussi certaines caractéristiques de l'évolution passée et présente de la participation du Québec à l'enseignement universitaire en distinguant les secteurs anglophone et francophone.

\section{TAUX DE PARTICIPATION}

Que signifient les taux provinciaux de participation à l'enseignement postsecondaire rapportés dans le rapport du Secrétariat d'Etat? Telle est la question fondamentale soulevées par les résultats des tableaux 2.1 et 2.2 de ce rapport ${ }^{3}$ (reproduits ici au tableau 1).

\section{Taux total de participation}

Selon le tableau 2.1, le Québec a le plus haut taux total de participation à l'enseignement postsecondaire au Canada, soit $27,4 \% .^{4}$ Qui plus est, il apparait clairement - à la comparaison de la répartition provinciale des inscriptions postsecondaires canadiennes et de la répartition provinciale de la population canadienne de 18 à 24 ans - que c'est seulement au Québec - et, à un degré moindre, en Ontario - que le taux d'inscription dépasse celui de la population des 18 à 24 ans; partout ailleurs au Canada, le taux d'inscription est relativement moins élevé, principalement dans les provinces de l'Atlantique et en Saskatchewan.

${ }^{1}$ Secrétariat d'Etat-Direction de l'aide à l'éducation. Quelques caractéristiques des étudiants du niveau postsecondaire au Canada, Ottawa, Thorn Press Limited, 1976.

2 Ibid., p. 2.

3 Ibid., p. 6 et p. 9.

${ }^{4}$ Le taux total de participation est le rapport entre le total des étudiants (à temps complet et à temps partiel) et la population des 18 à 24 ans. 
61 Remarques méthodologiques sur la participation

\section{IABLEFU I}

Taux global de participation et rëpartition des inscriptions et de la population des 18 à 24 ans par province, Canada $1974-75$. Type d'étudiant par province d'êtude, Canada, 1974-75.

Tableau 2.1 Taux total de particlpalion el rèpartition des inscriplions el do la populatlon des 18 a 24 ans par province, $1974-1975$

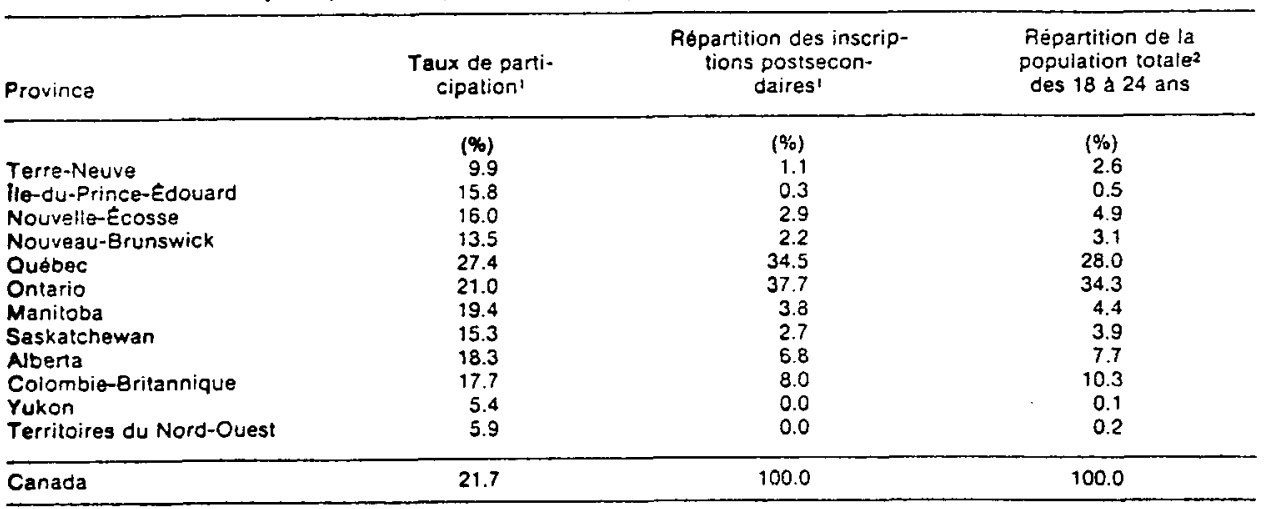

'Fondé sur lenquâte sur les étudiants du postsecondaire 1974-1975. Le taux de participation est le rappont entre le total des étudiants la temps preln et a temps partiel) at la population des 18 a 24 ans.

iFondé sur les estimations de Statistique Canada. Voir. Statistique Canada (1974).

Tableau 2.2 Type' d'étudiant par province d'étude

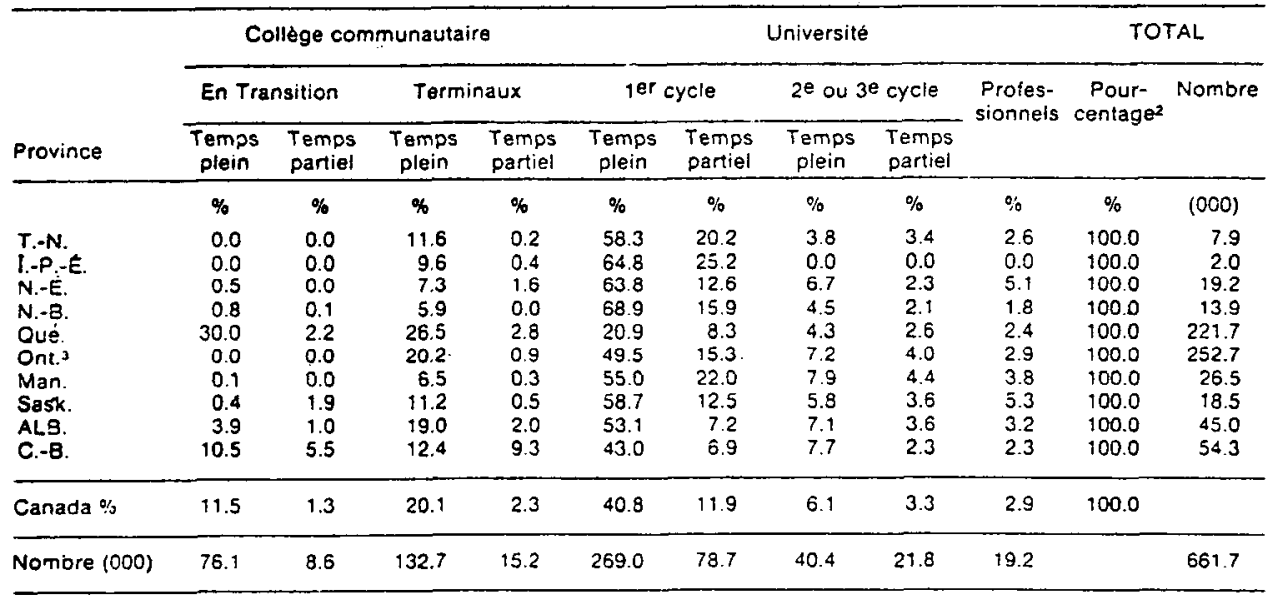

IYoir céfinitions à l'annexe I.

Le total ces chiffres arrondis peut différer de $100 \%$.

Bgien que le pourcentage des eltudiants en transition en Ontario soit de zèro, il pourraity avoir dans les colleges communautaires, des éluciants qui ont lintention de soursuivre leurs etudes a l'universite.

Source: Secrétariat d'Etat. Direction de l'aide à l'éducation. Quelques caractéristiques des étudiants du niveau postsecondaire au Canade, Otcawa 1976, p. 6 et p. 9.

\section{Taux de participation par province et par type d'étudiant}

Ces différences régionales apparaissent encore plus marquées au tableau 2.2 du rapport où est présentée la répartition "type d'étudiant par province d'étude". 
La différenciation régionale de la participation est néanmoins incomplète si n'est pas connue aussi la répartition "province d'étude par type d'étudiant". Nous avons calculé cette répartition à partir des pourcentages et des nombres totaux du tableau 2.2 du rapport et l'avons comparée à la répartition provinciale de la population des 18 à 24 ans et à la répartition provinciale des inscriptions postsecondaires canadiennes. Les résultats apparaissent aux tableaux $2 \mathrm{a}, 2 \mathrm{~b}$ et $2 \mathrm{c}$.

Les principales conclusions que l'on peut tirer sont les suivantes:

a) La prépondérance relative du Québec dans la répartition totale de l'effectif postsecondaire canadien par rapport au groupe d'âge témoin des 18 à 24 ans (34,5\% vs $28 \%$ ) n'est pas uniforme à tous les niveaux du postsecondaire ou d'un type d'étudiant à l'autre: cette prépondérance d'ensemble n'est due en fait qu'à une très forte représentation aux niveaux "collège communautaire en transition" $(86 \%)$ et "collège communautaire terminal" (44\%); il y a sous-représentation à tous les paliers du niveau universitaire. (Tableau $2 \mathrm{a}$ et $2 \mathrm{~b}$ )

b) A l'inverse du Québec, l'Ontario, absente au niveau "collège communautaire en transition", accroît sa prépondérance aux autres niveaux: "collège communautaire terminal" (36\%), ler cycle $(49 \%)$, 2e et $3 \mathrm{e}$ cycles (46\%). Les inscriptions à temps complet et à temps partiel à chacun de ces niveaux sont par ailleurs plus également réparties. (Tableau $2 b$ )

c) Alors que le tableau 2.1 du rapport n'indiquait, pour chacune des autres provinces, qu'une sous-représentation de l'effectif postsecondaire total par rapport au groupe des 18 à 24 ans, la comparaison par type d'étudiant permet maintenant d'apporter plus de nuances:

- Au niveau collégial, la sous-représentation par rapport au groupe des 18 à 24 ans est encore plus forte que pour l'ensemble du postsecondaire. (Tableau 2a)

- Cependant, au niveau universitaire, la sous-représentation est généralement moindre ou, pour certaines régions, fait place à une sur-représentation. C'est ainsi que chez le groupe à temps complet de niveau universitaire, quatre provinces sont légèrement sur-représentées, quatre autres légèrement sous-représentées. Chez le groupe à temps partiel de niveau universitaire, la sousreprésentation se maintient davantage, car seul le Manitoba est sur-représenté. (Tableau 2c)

d) $\mathrm{Si}$ on compare les représentations relatives des régions au premier cycle et au $2 \mathrm{e}$ et $3 \mathrm{e}$ cycles, les éléments suivants ressortent: la sous-représentation des Provinces de l'Atlantique est plus élevée au 2 e et $3 e$ cycles qu'au ler cycle (respectivement $9,8 \%$ vs $11,1 \%$ et $5,2 \%$ vs $11,1 \%$ ); par contre dans les régions à l'ouest de l'Ontario, on note moins de différences entre le ler cycle et le $2 \mathrm{e}$ et $3 \mathrm{e}$ cycles, et la représentation à chacun de ces niveaux, exception faite de la Colombie-Britannique, correspond généralement à l'importance relative du groupe des 18 à 24 ans. (Tableau 2b)

e) Retenons enfin, pour revenir au Québec, que les proportions qui s'approchent le plus de la proportion québécoise des 18 à 24 ans (28\%) sont celles qui correspondent aux types d'étudiants suivants:

- l'ensemble des effectifs à temps partiel à tous les niveaux, avec 28,4\% (Tableau 2c);

- l'effectif à temps partiel de $2 \mathrm{e}$ et $3 \mathrm{e}$ cycles, avec $26,7 \%$ (Tableau 2b);

- le regroupement comprenant toutes les inscriptions à temps complet de niveau 
TABLEAU 2a

Province d'ëtude par type d'étudiant du niveau postsecondaire Canada 1974-75 - Estimation (\%)*

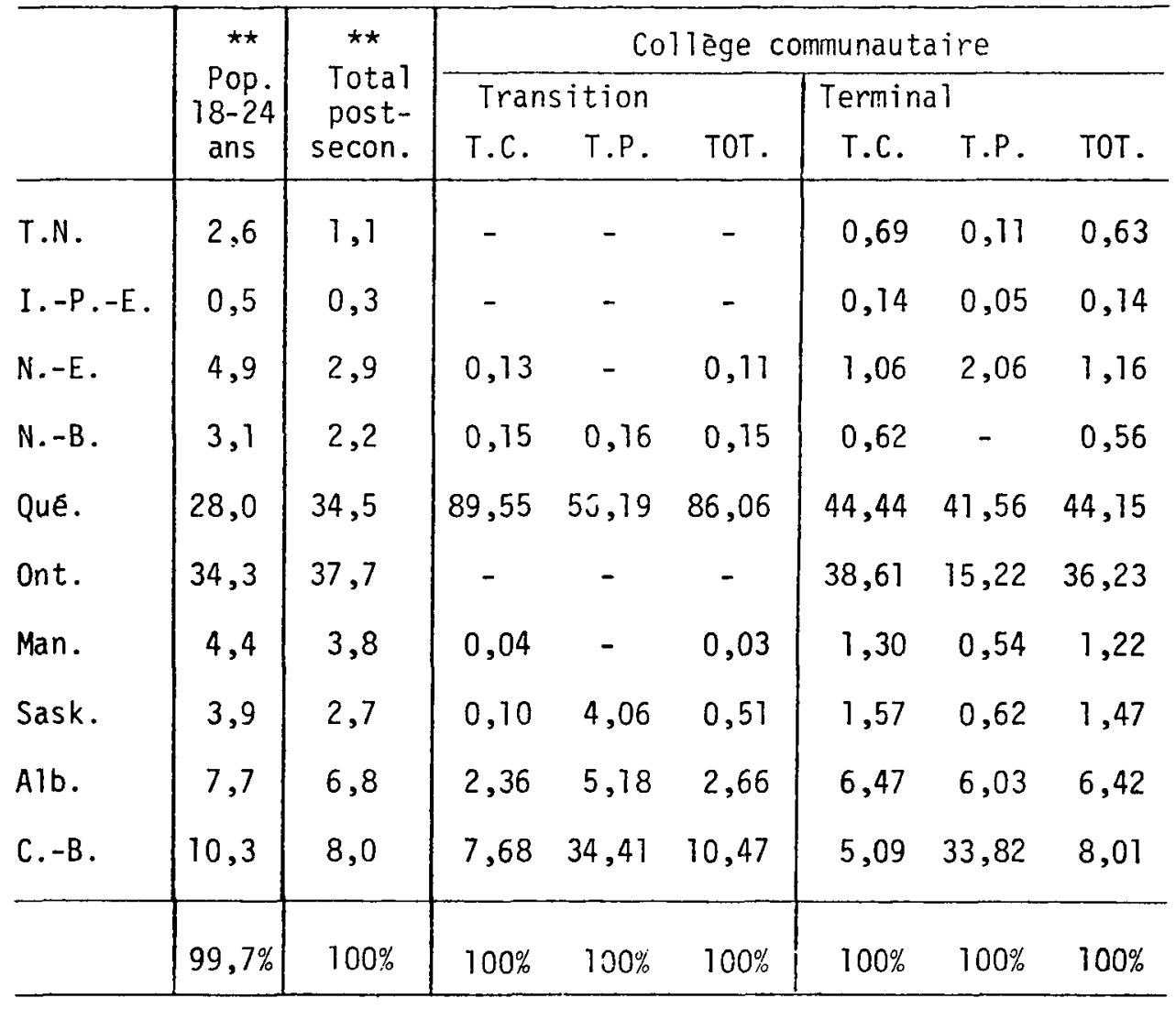

* Source: Estimation calculée à partir des pourcentages et des nombres totaux du tableau 2.2 in Secrétariat d'Etat Direction de l'aide à l'êducation, Quelques caractéristiques...., p. 9.

Source: Ibid, tableau 2.1, p. 6

Notes: $\quad$ T.C.: temps complet T.P.: temps partiel

universitaire et de niveau "collège communautaire en transition", avec 31,5\%.

(Tableau 2c)

\section{REMARQUES MÉTHODOLOGIQUES}

Afin d'éclairer les comparaisons entre le Québec et les autres régions (et aussi les comparaisons des autres régions entre elles) que proposent les résultats du sondage du 
TABLEAU 2b

Province d'étude par type d'étudiant du niveau postsecondaire - Canada $1974-75$ - Estimation $(\%)$ *

\begin{tabular}{|c|c|c|c|c|c|c|c|c|}
\hline \multirow{3}{*}{$\begin{array}{l}\text { Province } \\
\text { d'êtude }\end{array}$} & \multirow{3}{*}{$\begin{array}{l}\star \star \\
\text { Pop. } \\
18-24 \\
\text { ans }\end{array}$} & \multirow{3}{*}{$\begin{array}{c}\star \star \\
\text { Total } \\
\text { post- } \\
\text { secon. }\end{array}$} & \multicolumn{6}{|c|}{ UNIVERSITE } \\
\hline & & & \multicolumn{3}{|c|}{ premier cycle } & \multicolumn{3}{|c|}{$2 \mathrm{e}$ et $3 \mathrm{e}$ cycles } \\
\hline & & & T.C. & T.P. & TOT. & T.C. & T.P. & TOT. \\
\hline T. $-N$. & 2,6 & 1,1 & 1,69 & 2,02 & 1,77 & 0,74 & 1,25 & 0,92 \\
\hline I. - P. $-E$. & 0,5 & 0,3 & 0,48 & 0,64 & 0,51 & - & - & - \\
\hline N. $-E$. & 4,9 & 2,9 & 4,51 & 3,07 & 4,18 & 3,18 & 2,04 & 2,78 \\
\hline N. - B. & 3,1 & 2,2 & 3,52 & 2,80 & 3,36 & 1,54 & 1,35 & 1,48 \\
\hline Qué. & 28,0 & 34,5 & 17,05 & 23,31 & 18,46 & 23,55 & 26,71 & 24,65 \\
\hline Ont. & 34,3 & 37,7 & 46,02 & 48,99 & 46,69 & 44,95 & 46,85 & 45,61 \\
\hline Man. & 4,4 & 3,8 & 5,35 & 7,39 & 5,82 & 5,17 & 5,40 & 5,25 \\
\hline Sask. & 3,9 & 2,7 & 4,00 & 2,93 & 3,76 & 2,65 & 3,09 & 2,80 \\
\hline Alb. & 7,7 & 6,8 & 8,79 & 4,11 & 7,74 & 7,89 & 7,57 & 7,76 \\
\hline \multirow[t]{2}{*}{ C. $-B$. } & 10,3 & 8,0 & 8,59 & 4,75 & 7,73 & 10,33 & 5,79 & 8,75 \\
\hline & $99,7 \%$ & $100 \%$ & $100 \%$ & $100 \%$ & $100 \%$ & $100 \%$ & $100 \%$ & $100 \%$ \\
\hline
\end{tabular}

* Source: Estimation calculée à partir des pourcentages et des nombres totaux du tableau 2.2 in Secrétariat d'Etat - Direction de l'aide à l'êducation, Quelques caractéristiques ..., p. 9

Source: Ibid, tableau 2.1, p. 6

Notes: T.C.: temps complet T.P.: temps partiel

Secrétariat d'Etat, il importe aussi de faire quelques remarques méthodologiques. Nous n'en retiendrons ici que certaines qui ont trait à l'âge modal de passage aux étudues postsecondaires, à la durée des études collégiales, à la durée des études de premier cycle et au lieu de résidence des étudiants.

\section{Age modal de passage aux études postsecondaires}

Les études postsecondaires au Canada débutent généralement après la $12 \mathrm{e}$ année d'études, sauf en Ontario où elles débutent après la $13 \mathrm{e}$ année et à Terre-Neuve où elles débutent après la 11e année. (Sauf aussi au Québec où elles débutent maintenant après la $11 \mathrm{e}$ 
TABLEAU 2c

Province par type d'étudiant du niveau postsecondaire - Canada $1974-75$ - Estimation $(\%) *$

\begin{tabular}{|c|c|c|c|c|c|c|c|c|}
\hline \multirow{2}{*}{$\begin{array}{l}\text { Province } \\
\text { d'êtude }\end{array}$} & \multirow{2}{*}{$\begin{array}{l}\star \star \\
\text { Pop } \\
18-24 \\
\text { ans }\end{array}$} & \multirow{2}{*}{$\begin{array}{c}* \star \\
\text { Total } \\
\text { post- } \\
\text { secon. }\end{array}$} & \multicolumn{2}{|c|}{$\begin{array}{l}\text { Université } \\
+ \text { collège } \\
\text { communautaire }\end{array}$} & \multicolumn{3}{|c|}{ Université } & \multirow{2}{*}{$\begin{array}{l}\text { Université } \\
\text { T.C. + } \\
\text { Transition } \\
\text { T.C. }\end{array}$} \\
\hline & & & T.C. & T.P. & T.C. & T.P. & TOT. & \\
\hline T. $-N$. & 2,6 & 1,1 & 1,12 & 1,51 & 1,54 & 1,86 & 1,62 & 1,26 \\
\hline I. - P. $-E$. & 0,5 & 0,3 & 0,28 & 0,41 & 0,39 & 0,50 & 0,42 & 0,32 \\
\hline N. $-E$. & 4,9 & 2,9 & 2,98 & 2,55 & 4,38 & 2,85 & 4,03 & 3,60 \\
\hline N.B. & 3,1 & 2,2 & 2,12 & 2,03 & 3,16 & 2,49 & 3,00 & 2,61 \\
\hline Qué. & 28,0 & 34,5 & 34,69 & 28,40 & 18,48 & 24,04 & 19,78 & 37,50 \\
\hline Ont. & 34,3 & 37,7 & 37,51 & 41,13 & 45,49 & 48,55 & 46,20 & 37,15 \\
\hline Man. & 4,4 & 3,8 & 3,61 & 5,70 & 5,34 & 6,96 & 5,72 & 4,37 \\
\hline Sask. & 3,9 & 2,7 & 2,80 & 2,76 & 3,90 & 2,96 & 3,68 & 3,20 \\
\hline Alb. & 7,7 & 6,8 & 7,22 & 5,00 & 8,62 & 4,83 & 7,74 & 7,47 \\
\hline \multirow[t]{2}{*}{ C. $-B}$. & 10,3 & 8,0 & 7,67 & 10,50 & 8,69 & 4,97 & 7,82 & 8,51 \\
\hline & $99,7 \%$ & $100 \%$ & $100 \%$ & $100 \%$ & $100 \%$ & $100 \%$ & $100 \%$ & $100 \%$ \\
\hline
\end{tabular}

* Source: Estimation calculée à partir des pourcentages et des nombres totaux du tableau 2,2 in Secretariat d'Etat. Direction de 1 'aide à l'éducation, Quelques caractéristiques ..., p. 9

Source: Ibid, tableau 2.1, p.6

Notes: T.C.: temps complet T.P.: temps partiel

Transition T.C.: collège communautaire en transition - temps complet année, bien qu'au moment de l'enquête, en 1974-75, les nouveaux inscrits de niveau collégial étaient censés avoir fait leur $12 \mathrm{e}$ année.)

\section{Durée des programmes d'études collégiaux}

Les programmes collégiaux de transition menant aux études universitaires ont généralement une durée de deux ans au Québec alors que cette durée est variable ailleurs au Canada (un an ou deux); les programmes collégiaux terminaux ou professionnels ont au Québec une 
durée de trois ans alors que cette durée ailleurs au Canada est généralement variable (deux, trois ou quatre ans). Sans que l'on soit en mesure de quantifier ce facteur, on serait néanmoins justifié de supposer qu'il explique, en partie, le grossissement de l'effectif collégial du Québec par rapport à ceux du reste du Canada.

\section{Durée des études de premier cycle}

La durée des études de premier cycle est de trois ans au Québec alors qu'elle est le plus souvent de quatre années ailleurs au Canada. A l'inverse du précédent, ce facteur aurait un effet négatif sur la mesure de la participation du Québec aux études de premier cycle.

\section{Lieu de résidence des étudiants}

A l'examen des données du tableau AV-6 du rapport ${ }^{5}$ qui présente la répartition des étudiants selon la province de résidence et la province d'étude, on est en mesure d'affirmer que le fait d'établir les taux de participation à l'enseignement postsecondaire à partir des inscriptions dans la province d'étude peut faire en sorte, à des degrés divers, de sousestimer, dans l'ordre, le taux de participation total des résidents de l'Ile-du-Prince-Edouard, de Terre-Neuve, de la Saskatchewan, du Nouveau-Brunswick et du Québec et de surestimer, dans l'ordre, le taux de participation total des résidents de la Nouvelle-Ecosse, de l'Ontario et de l'Alberta.

Ces remarques, rappelons-le ici, ne visent en rien à porter un jugement négatif sur le sondage du Secrétariat d'Etat dont l'objectif premier, d'ailleurs, n'était pas de mesurer fidèlement des taux de participation réels à l'enseignement postsecondaire mais plutôt d'en décrire et d'en analyser les modalités selon diverses variables socio-économiques. Ces remarques visent uniquement à rappeler que le terme "enseignement postsecondaire" n'a pas le même sens partout au Canada; que le nombre de cohortes d'étudiants qu'il comporte n'est pas nécessairement le même d'une province à l'autre ou d'un système d'éducation à l'autre; et, qu'en conséquence, l'utilisation qu'on peut faire des chiffres officiels ou de résultats d'enquête doit toujours être circonstanciée. On comprendra aisément, par ailleurs, qu'au moment où les effets de la réforme des structures scolaires du Québec commencent à se faire sentir au niveau postsecondaire collégial et commenceront très bientôt à se faire sentir au niveau postsecondaire universitaire, l'on soit intéressé à établir une mesure la plus juste et la plus fidèle possible de la participation réelle du Québec à l'enseignement postsecondaire et de l'évolution de cette participation.

\section{QUELQUES CARACTÉRISTIQUES PARTICULIĖRES DE LA PARTICIPATION DU QUÉBEC AU NIVEAU UNIVERSITAIRE}

C'est pour éclairer ce phénomène que nous brosserons maintenant, à grands traits, l'évolution de la participation du Québec à l'enseignement postsecondaire - surtout universitaire - depuis les 10 ou 15 dernières années. Nous utiliserons, pour ce faire, les données des recensements de 1961 et 1971 ainsi que certaines conclusions des travaux menés depuis trois ans par M. Michel Robillard, ${ }^{6}$ démographe au Service de la recherche

${ }^{5}$ Ibid., p. 138

${ }^{6}$ Michel Robillard, Les Clientèles universitaires au Québec: évolution passée et perspectives d'avenir 1966-1990, Université du Québec, (1976). 
institutionnelle de l'Université du Québec. Dans cette analyse, nous distinguerons les secteurs universitaires anglophone et francophone.

\section{Fréquentation et scolarisation universitaires au Québec de 1961 à 1971}

En 1961, selon les données du Recensement, 6,12\% de la population québécoise de plus de 15 ans fréquentait à temps complet une institution de niveau universitaire, ${ }^{7}$ au moment du recensement, ou déclarait avoir atteint un tel niveau de scolarité, dans le passé (Tableau 3). En Ontario et dans les autres provinces canadiennes, les pourcentages étaient respectivement $6,55 \%$ et $6,68 \%$, soit dans ce dernier cas, un taux supérieur de $9 \%$ à la moyenne québécoise. En 1971, ce léger écart ne s'était guère atténué puisque $9,81 \%$ des québécois de 15 ans et plus poursuivaient à temps complet des études universitaires ou avaient atteint ce niveau de scolarité; dans les autres provinces, $11,22 \%$ appartenaient à cette catégorie, soit un taux supérieur de 14\% à la moyenne québécoise. En 1961 et 1971, on retrouvait donc au Québec une très légère sous-scolarisation qui, somme toute, ne démontrait pas un retard profond de la population québécoise dans son ensemble.

Considérée sur la base des groupes linguistiques cependant, l'analyse de la fréquentation et de la scolarisation universitaires au Québec présente une toute autre image. Selon les données du recensement de 1971, la fréquentation et la scolarisation universitaires des francophones $(7,78 \%)$ apparaissaient comme étant près de trois fois inférieures à celles des anglosphones $(20,05 \%)$ et près de deux fois inférieures à celles des allophones $(13,48 \%)$. Par contre, le niveau de fréquentation et de scolarisation universitaires des anglophones du Québec $(20,05 \%)$ était près de deux fois supérieur à celui des Canadiens des autres provinces $(11,22 \%)$.

\section{Evolution de la fréquentation universitaire au Québec dans les secteurs francophone et anglophone, de 1966 à 1975}

L'analyse de l'évolution des taux de fréquentation universitaire des secteurs anglophone et francophone depuis 1966 permet d'évaluer dans quelle mesure le système francophone se sera "rattrapé". L'évolution actuelle et future de ces taux est intimement liée aux changements de structures scolaires survenus au Québec depuis le milieu de la décennie '60. Rappelons-en les principales caractéristiques.

\section{Les changements de structures scolaires}

Avant le réaménagement destructures scolaires québécoises au niveau collégial, le système scolaire anglophone, de type nord-américain, favorisait davantage l'accès à l'université que le système scolaire francophone, d'inspiration européenne. La différence principale résidait dans la durée des études pré-universitaires et l'âge d'accès à l'université, inférieurs d'au moins deux ou trois ans dans le système anglophone. Cette différence pouvait expliquer, en partie, l'écart de scolarisation universitaire entre les deux secteurs linguistiques.

Les nouvelles structures scolaires québécoises ont le mérite d'uniformiser les cheminements scolaires des systèmes anglophone et francophone. Dans le système anglophone, le réaménagement des strutures s'est effectué entre 1970 et 1973: il a consisté principalement en l'implantation d'une structure collégiale, alors inexistante, qui a eu pour

${ }^{7}$ Au recensement le niveau universitaire correspond à toute année de scolarité au-delà de la 13e année excluant l'enseignement technique et professionnel. 
TABLEAU 3

Fréquentation et scolarisation universitaires: population de 15 ans et plus, Quēbec, Ontario, Canada sans Quêbec, 1961 et 1971, et Québec selon 1a langue maternelle, 1971.

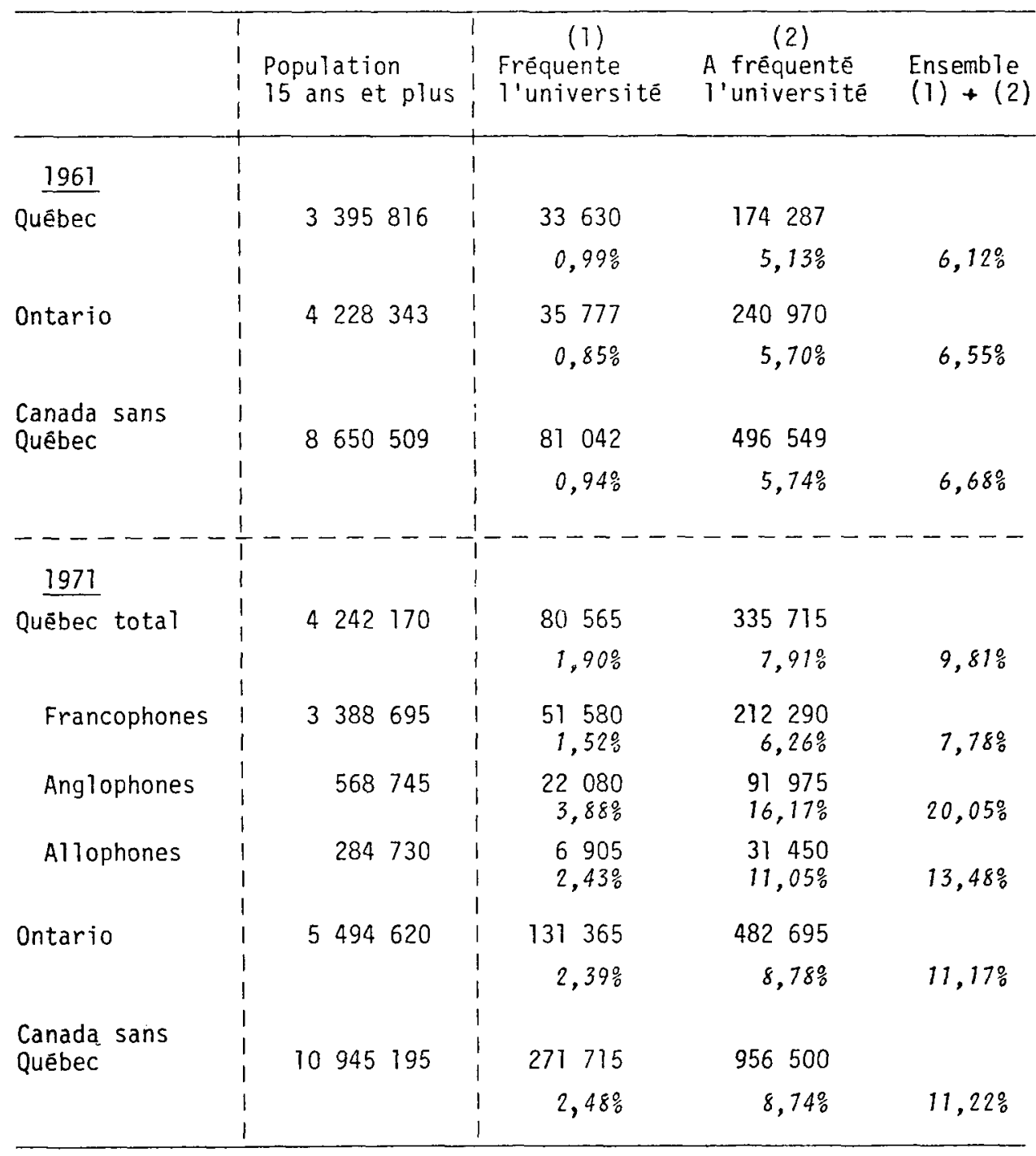

Source: Statistique Canada. Recensements de 1961 et 1971.

effet d'augmenter de deux ans la durée des études pré-universitaires, de retarder ainsi l'âge d'entrée à l'université - qui est passé de 17 à 19 ans -, et de ramener de quatre à trois ans la durée des programmes de premier cycle. L'impact de cette transformation sur le taux de fréquentation pourrait être négatif. Dans le système francophone, ce sont le réaménagement 
du niveau collégial, la disparition des écoles normales et l'intégration de la formation des maîtres à l'université, puis l'élimination de la 7ième année à l'élémentaire, en 1973, qui auront un effet positif sur le taux de fréquentation universitaire en réduisant la durée des études pré-universitaires et l'âge d'entrée à l'université. Comparativement à l'étudiant francophone du début des années '60 qui entrait à l'université à 21 ans, celui d'aujourd'hui y entrera désormais à 19 ans.

Aux effets de fond de ces changements de structure se sont ajoutés (et s'ajouteront) des effets temporaires sur le volume des inscriptions: on a observé un gonflement temporaire des inscriptions dans les universités francophones, entre 1968 et 1971, dû à l'arrivée de doubles promotions du secteur collégial; il y a eu réduction temporaire des inscriptions dans les universités anglophones, de 1970 à 1973, en raison du retard d'entrée à l'université des finissants du niveau secondaire; enfin, il faut s'attendre à un gonflement des inscriptions dans les universités francophones, entre 1978 et 1981 surtout, qui traduira le phénomène de double promotion occasionné par la suppression de la 7 ième année, en 1973.

\section{La fréquentation universitaire à temps complet}

Pour décrire véritablement l'évolution de la fréquentation universitaire à temps complet, au Québec, au cours des dix dernières années, il faut donc tenir compte de ces changements de structure du système scolaire survenu depuis le milieu de la décennie ' 60 . C'est ainsi qu'on a été amené à développer des taux réels de fréquentation - par opposition à des taux apparents. Le taux réel de fréquentation est le rapport à la population des 18 à 24 ans des inscriptions universitaires de premier cycle, corrigées pour tenir compte, par exemple, de facteurs tels les inscriptions de niveau universitaire dans les écoles normales, la transformation des structures aux niveaux universitaire et collégial et, en particulier, la durée des études universitaires et pré-universitaires.

De 3,5 pour cent qu'il était, en 1966, le taux réel de fréquentation universitaire dans le secteur francophone est passé à 5,69 pour cent, en 1975, augmentant ainsi à un rythme annuel moyen de $5,5 \%$. Toutefois, malgré cette amorce de rattrapage, la fréquentation universitaire des francophones demeurait encore, en 1975, près de trois fois inférieure à celle des anglophones du Québec (16,9 pour cent), malgré que cette dernière eut connu un rythme annuel moyen d'augmentation limité à 2,6\%, depuis 1966. (Tableau 4)

\section{La fréquentation universitaire à temps partiel}

Comme il est très difficile, au temps partiel, d'évaluer quel a pu être l'impact du réaménagement des structures d'enseignement sur le volume des inscriptions, seule l'utilisation des taux de fréquentation apparents aura permis d'établir le rapport des inscriptions à temps partiel de premier cycle à la population des 20 à 39 ans.

Dans le secteur francophone, ce taux est passé de 3,8 pour mille, en 1966, à 22,5 pour mille, en 1975 , soit une augmentation annuelle moyenne de $20 \%$; dans le secteur anglophone, il est passé, pour la même période, de 36,5 à 50,5 pour mille, soit une augmentation annuelle moyenne de $3,7 \%$. (Tableau 4)

\section{Structure d'âge dans les secteurs universitaires anglophone et francophone du Québec}

Un autre indice, enfin, permet également de décrire les différences entre les deux secteurs 


\section{TABLEAU 4}

Taux de frêquentation et structure d'âge: secteurs universitaires francophone et anglophone du Québec. Annēes choisies entre 1969 et 1975.

Taux de frêquentation (ler cycle):

- temps complet/

pop. $18-24$ ans

1966

3,57

13,3

1975

5,69

16,9

$\triangle$ annuel

moyen

$5,5 \%$

$2,6 \%$

- temps partiel/

$\frac{\text { pop. } 20-39 \text { ans }}{\text { (pour 1000)** }}$

1966
1969
1975
$\Delta$ annuel
moyen

36,5

(pour 1000)**

44,6

7,42

50,5

22,5

$3,7 \%$

Structure d'âge (ler cycle):

- temps complet

$\begin{array}{llll}\text { moins de } 25 \text { ans } & 1974 & 85,9 \% & 87,8 \% \\ & 1975 & 85,0 \% & 86,2 \% \\ \text { moins de 20 ans } & 1974 & 13,2 \% & 26,0 \% \\ & 1975 & 11,8 \% & 22,0 \%\end{array}$

- temps partiel (1975)

$\begin{array}{lll}20-24 \text { ans } & 20,2 \% & 28,7 \% \\ 25-29 \text { ans } & 30,5 \% & 30,5 \% \\ 30-34 \text { ans } & 23,9 \% & 16,7 \% \\ 35 \text { ans et plus } & 23,8 \% & 22,8 \%\end{array}$

Source: Michel Robillard, Les clientèles universitaires au Québec:

Évolution passēe et perspectives d'avenir 1966-1990. Université du Québec, (1976), tableau 31, p. 154 et tableau 3, p. 25.

* Taux global réel de fréquentation pour 100.

** Taux global apparent de fréquentation pour 1000. 
universitaires québécois: la structure d'âge. Ses grandes caractéristiques, rapportées au Tableau 4, sont les suivantes:

- Au temps complet premier cycle, dans chaque secteur, environ $85 \%$ des étudiants ont moins de 25 ans. Cependant, près de deux fois plus d'étudiants du secteur anglophone ont moins de 20 ans; on note toutefois que la proportion des moins de 20 ans a baissé considérablement, en 1975, dans les universités anglophones. Le réaménagement des structures de programmes de premier cycle dans les universités anglophones aura peut-être comme conséquence d'augmenter l'âge moyen des étudiants et de rapprocher leur structure d'âge de celle des étudiants des universités francophones.

Au temps partiel, premier cycle, les étudiants sont beaucoup mieux répartis entre les divers groupes d'âge. Toutefois, dans les universités francophones, la structure d'âge est légèrement différente et la clientèle, légèrement plus âgée; dans les universités anglophones, le groupe des 20 à 24 ans est proportionnellement moins nombreux et celui des 30 à 34 ans, proportionnellement plus nombreux.

\section{CONCLUSION}

Le sondage du Secrétariat d'Etat a permis assurément de rassembler une masse imposante de données, pour la plupart utilisables. Cependant, compte tenu des possibilités qu'offre le traitement de ces données, on se serait peut-être attendu, d'un point de vue québécois, à une analyse plus détaillée des différentes caractéristiques qui aurait privilégié la variable régionale et départagé davantage les types d'étudiants. Heureusement, les données demeurent disponibles et leur analyse pourra toujours être poursuivie en ce sens.

Par ailleurs l'absence de la variable linguistique ne donne pas au rapport toute la signification qu'il aurait pu avoir pour le Québec. A partir de la question du questionnaire du sondage portant sur le nom de l'établissement, il serait toutefois aisé de reprendre ou poursuivre l'analyse en distinguant les secteurs anglophone et francophone au Québec.

Dans les études canadiennes et la statistique officielle portant sur la participation régionale à l'enseignement postsecondaire, il faudrait pouvoir distinguer les niveaux collégial et universitaire; il faudrait de plus, dans le cas du Québec, retenir la variable linguistique, essentielle à la description et à l'analyse de l'évaluation de l'enseignement supérieur. 